\title{
The Reflection Grating Spectrometer on board XMM-Newton
}

\author{
J. W. den Herder ${ }^{1}$, A. C. Brinkman ${ }^{1}$, S. M. Kahn ${ }^{2}$, G. Branduardi-Raymont ${ }^{3}$, K. Thomsen ${ }^{4}$, H. Aarts ${ }^{1}$,
} M. Audard ${ }^{4}$, J. V. Bixler 6 , A. J. den Boggende ${ }^{1}$, J. Cottam ${ }^{2}$, T. Decker ${ }^{6}$, L. Dubbeldam ${ }^{1}$, C. Erd $^{5}$, H. Goulooze ${ }^{1}$, M. Güdel ${ }^{4}$, P. Guttridge ${ }^{3}$, C. J. Hailey ${ }^{2}$, K. Al Janabi ${ }^{3}$, J. S. Kaastra ${ }^{1}$, P. A. J. de Korte ${ }^{1}$, B. J. van Leeuwen ${ }^{1}$, C. Mauche ${ }^{6}$, A. J. McCalden ${ }^{3}$, R. Mewe ${ }^{1}$, A. Naber ${ }^{1}$, F. B. Paerels ${ }^{2}$, J. R. Peterson ${ }^{2}$, A. P. Rasmussen ${ }^{2}$, K. Rees ${ }^{3}$, I. Sakelliou ${ }^{3}$, M. Sako 2 , J. Spodek ${ }^{2}$, M. Stern ${ }^{2}$, T. Tamura ${ }^{1}$, J. Tandy $^{3}$, C. P. de Vries ${ }^{1}$, S. Welch ${ }^{3}$, and A. Zehnder ${ }^{4}$

1 Space Research Organization of The Netherlands, Sorbonnelaan 2, 3584 CA Utrecht, The Netherlands

2 Columbia Astrophysics Laboratory, Columbia University, 550 West 120th Street, New York, NY 10027, USA

3 Mullard Space Science Laboratory, University College London, Holmbury St. Mary, Dorking, Surrey, RH5 6NT, UK

4 Paul Scherrer Institute, 5232 Villigen, Switzerland

5 Astrophysics Division, Space Science Department of ESA, ESTeC, 2200 AG Noordwijk, The Netherlands

${ }^{6}$ Lawrence Livermore National Laboratory, PO Box 808 Livermore, CA 94550, USA

Received 2 October 2000 / Accepted 26 October 2000

\begin{abstract}
The ESA X-ray Multi Mirror mission, XMM-Newton, carries two identical Reflection Grating Spectrometers (RGS) behind two of its three nested sets of Wolter I type mirrors. The instrument allows highresolution $(E / \Delta E=100$ to 500$)$ measurements in the soft X-ray range (6 to $38 \AA$ or 2.1 to $0.3 \mathrm{keV})$ with a maximum effective area of about $140 \mathrm{~cm}^{2}$ at $15 \AA$. Its design is optimized for the detection of the K-shell transitions of carbon, nitrogen, oxygen, neon, magnesium, and silicon, as well as the L shell transitions of iron. The present paper gives a full description of the design of the RGS and its operational modes. We also review details of the calibrations and in-orbit performance including the line spread function, the wavelength calibration, the effective area, and the instrumental background.
\end{abstract}

Key words. space vehicles: instruments, reflection gratings, XMM-Newton

\section{Introduction}

The 0.2 to $10 \mathrm{keV}$ energy band contains the K-shell transitions of carbon, nitrogen, oxygen, neon, magnesium, silicon, sulphur and calcium, as well as both the L and $\mathrm{K}$ shell transitions of iron. Of particular interest, due to their high abundances, are oxygen and iron at temperatures of $10^{6} \mathrm{~K}$ and $10^{7}-10^{8} \mathrm{~K}$, respectively. Detailed analysis of spectral features from these elements will permit the determination of density, temperature, ionisation state, elemental abundances, mass motions and velocity shifts of the emitting regions and their surrounding environments. The spectral properties of the medium will depend on the excitation process (collisional or photo-ionization and recombination), the state (transient or quiescent) and on whether the plasma is optically thick or thin. With RGS it is possible to measure most of these transitions with the notable exception of those associated with the Fe Kshell. Amongst others, observations with RGS will give new information about the heating of stellar coronae, the

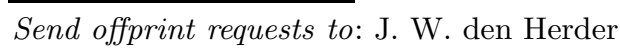

propagation of supernova remnant shocks through the interstellar medium, the conditions in the circumsource environments of X-ray binaries and active galactic nuclei, and the hot intracluster medium of clusters of galaxies.

\section{Instrument description}

\subsection{Optical design}

There are two identical RGSs behind two of the three mirror assemblies. The RGS design is illustrated in Fig. 1. Each incorporates an array of reflection gratings placed in the converging beam of the XMM-Newton telescope. This telescope, of type Wolter I, has a focal length of $7500 \mathrm{~mm}$ and includes 58 nested shells (Jansen et al. 2001). The grating stack consists of 182 precisely aligned reflection gratings and intercepts about half of the light emanating from the telescope. The undeflected light passes through and is intercepted by EPIC in the telescope focal plane. The gratings are actually located on a toroidal Rowland surface, formed by rotating the Rowland circle about an axis passing through the telescope and spectroscopic foci, 


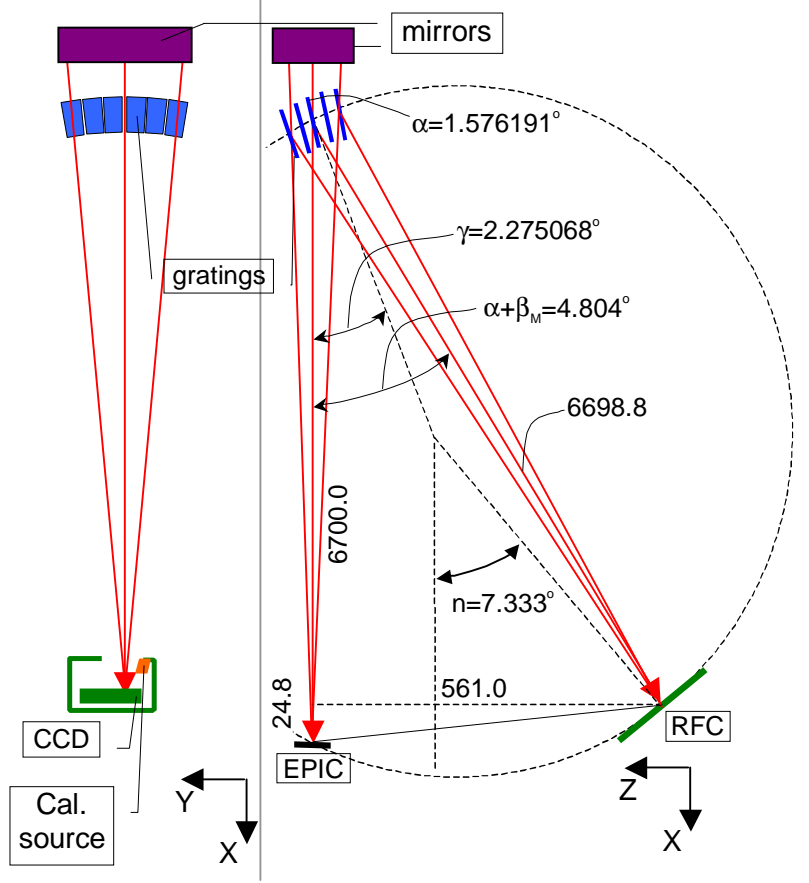

Fig. 1. Optical design of the RGS (not to scale). X-rays, indicated by red arrows, enter from the top. Numerical values for a few key dimensions and angles are indicated (linear dimensions in $\mathrm{mm}$, angles in degrees)

as illustrated in the left panel of Fig. 1. The gratings are slightly trapezoidal, since their edges lie along rays converging on the telescope focus. The field of view in the cross dispersion direction is determined by the width of the CCDs $( \pm 2.2$ arcmin), and the spatial resolution in this direction is largely determined by the imaging properties of the mirror. In the dispersion direction this is more complex: the extent of the source affects the wavelength resolution.

Nine large format back-illuminated CCDs are located on the Rowland circle to detect the dispersed spectra in single photon counting mode. The position of the X-ray on the detector gives the wavelength through the dispersion equation:

$m \lambda=d(\cos \beta-\cos \alpha)$,

where $m$ is the spectral order $(-1,-2, \ldots), d$ is the groove spacing and $\alpha$ and $\beta$ are the angles of the incident and dispersed rays, respectively, both measured from the grating plane. First, second and higher order spectra are overlapping on the detectors, however, they are easily separated using the inherent energy resolution of the CCDs. Key parameters of the design are listed in Table 1 (see also Brinkman et al. 1998).

\subsection{Reflection grating array}

The Reflection Grating Array (RGA) contains 182 identical gratings (one of the two RGAs contains 181 gratings due to a fabrication problem encountered during installation). The gratings are mounted at grazing incidence in
Table 1. Key design parameters

\begin{tabular}{|rc|}
\hline par & value comment \\
\hline$\lambda_{\text {blaze }}$ & $15 \AA$ A first order blaze wavelength \\
$\delta$ & $0.6989^{\circ}$ blaze angle of facets \\
$\gamma$ & $2.2751^{\circ}$ graze angle on facets \\
$\alpha$ & $1.5762^{\circ}$ angle of incidence \\
$\beta_{\text {blaze }}$ & $2.9739^{\circ}$ diffraction angle for $\lambda_{\text {blaze }}$ \\
$1 / d$ & 645.6 lines $/ \mathrm{mm}^{\text {Central groove density }}$ \\
CCDs & 9 back illuminated, $\sim 30 \mu \mathrm{m}$ thick \\
pixels & $27 \times 27 \mu \mathrm{m}^{2}$ pixel size; standard \\
& $3 \times 3$ on chip binned \\
CCD size & $1024 \times 384$ image area, identical storage area \\
$\mathrm{T}-80--120{ }^{\circ} \mathrm{C}$ nominally $-80^{\circ} \mathrm{C}$
\end{tabular}

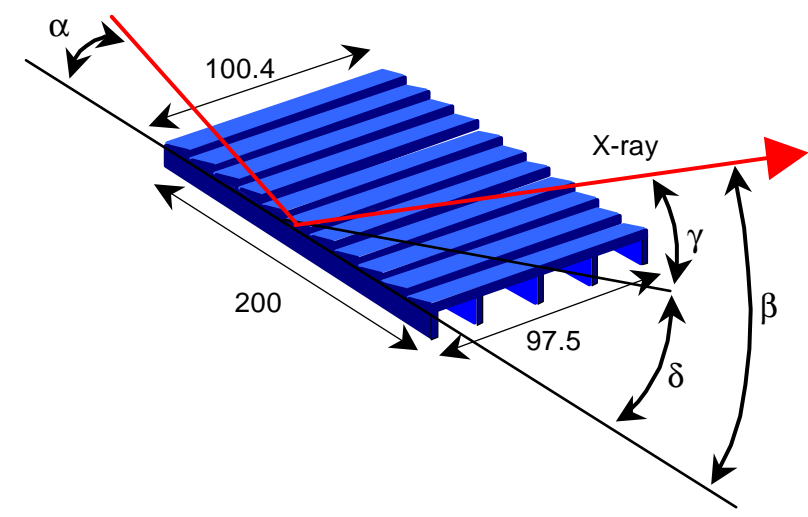

Fig. 2. Schematic drawing of a grating, including some of the key dimensions and angles

the in-plane, or classical diffracted X-rays lie in a plane that is perpendicular to the grating grooves. Because the beam is converging, the gratings are oriented so that the angle of the incident X-ray at the center of the grating, $\alpha$, is the same for all gratings in the array. In addition, the gratings all lie on the Rowland circle, which also contains the telescope focus and the spectroscopic focus for the blaze wavelength. In this configuration, aberrations, which would otherwise be introduced by the arraying, are eliminated (Kahn 1990) (see right side of Fig. 1). The telescope aperture is filled by rotating the Rowland circle about an axis passing through the telescope and spectroscopic blaze foci. In all, each RGA contains six rows of gratings.

Each grating measures about 10 by $20 \mathrm{~cm}$ (see Fig. 2). These large gratings need to be very flat in the long (i.e. dispersion) direction, since any non-flatness translates directly into a degradation of the resolution. At the same time the grating substrates need to be very thin in order to minimize the obstruction of the direct beam by the gratings. The grating substrates consist of $1 \mathrm{~mm} \mathrm{SiC}$ face sheets with five stiffening ribs at the back, running in the direction of the X-ray beam. The face sheets are fabricated to $1 \lambda(634.8 \mathrm{~nm})$ and $10 \lambda$ flatness in the long and the short direction, respectively. The gratings are 
replicated from a mechanically ruled master and are covered with a $2000 \AA$ gold coating. The groove density varies slightly $( \pm 10 \%)$ over the length of the gratings, to correct for aberrations associated with the converging beam (Hettrick \& Bowyer 1983; Kahn 1990). The groove density is $\sim 646$ grooves $/ \mathrm{mm}$ at the center.

The grating array support structure was machined out of a monolithic billet of vacuum hot-pressed beryllium. Beryllium was selected for the grating support structure for its low specific mass and good stability over the operational temperature range $\left(10-30{ }^{\circ} \mathrm{C}\right)$. This structure is mounted with three flexible kinematic mounts to the mirror support structure (Wilke et al. 1995).

To obtain the desired resolution, it is essential that all gratings are properly aligned (with $1 \mu \mathrm{m}$ tolerance on the position of any grating corner). This high precision alignment is achieved by positioning the individual gratings against four, coplanar bosses, which are precision machined into stainless steel rails. Half of the rails have been positioned using a high precision measurement device. The remaining half (those at the top) are roughpositioned, and then precision aligned interferometrically using the inserted gratings themselves as a reference. For a more detailed description of the RGAs see Kahn et al. (1996).

\subsection{Focal plane camera}

The spectrum is integrated on nine large format back illuminated CCDs. These nine CCDs are mounted in a row, following the curvature of the Rowland circle (see top two panels of Fig. 3). To reduce the dark current the CCDs are cooled to $-80{ }^{\circ} \mathrm{C}$. The increase in CCD charge transfer inefficiency (CTI) due to radiation damage can be reduced by lowering the temperature to $-120{ }^{\circ} \mathrm{C}$. Cooling is accomplished by a two-stage radiator, facing deep space, and by three nested thermal shells around the CCD bench (see Thomsen 1991). The first shield also contains four internal calibration sources. These consist of the $\alpha$ emitter ${ }^{244} \mathrm{Cm}$, and an $\mathrm{Al}$ target or a Teflon target, which produces $\mathrm{Al} \mathrm{K} \alpha(1487 \mathrm{eV})$ and $\mathrm{F} \mathrm{K} \alpha(676.8 \mathrm{eV})$ fluorescent emission. These sources each illuminate a small area of two CCDs, which is offset in the cross dispersion direction from the source image. In addition, the energies of the internal calibration sources are selected to be different from the energies (both orders) of the dispersed astrophysical spectra at the same position. At launch, the camera was protected by a door, which was opened in orbit (it can not be closed).

The nine CCD chips are back-illuminated GEC/EEV devices with an image and storage section of 384 by 1024 pixels each and a pixel size of $27 \times 27 \mu \mathrm{m}^{2}$. The width of the detector is matched to the size of the RGS spectrum in the cross dispersion direction. The length of the CCD assembly $(253 \mathrm{~mm})$ covers the first order $6-38 \AA$ wavelength range. The inter-chip gap between two adjacent CCDs is about $0.5 \mathrm{~mm}$. For optimum scientific performance, the
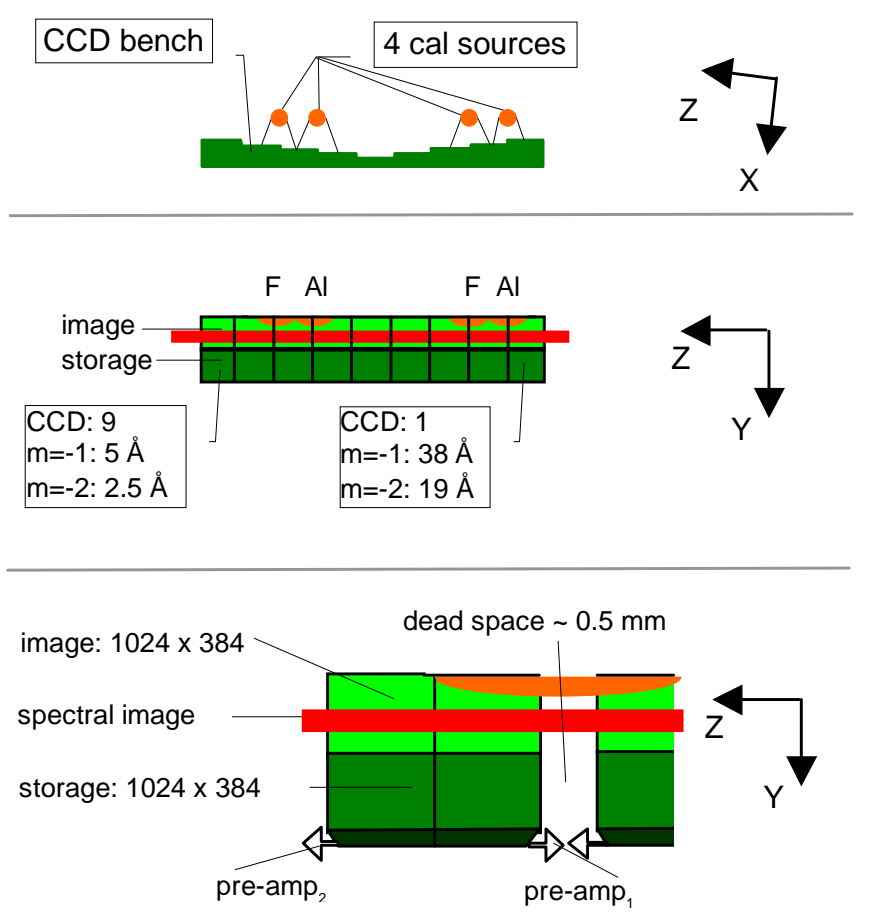

Fig. 3. CCD bench (top two panels) as well an enlarged view of two CCDs (bottom panel). The figure is not to scale

detector is operated in frame transfer mode; the image is first accumulated in one half of the CCD (the image section) and then quickly (20 msec) transferred to the other half (storage section), where it is read out through two low noise serial output registers. This doubles the read out speed and gives redundancy in case of an amplifier failure. The width of the line spread function (typically in the order of $250 \mu \mathrm{m}$ ) allows for $3 \times 3$ on-chip binning (OCB) of the pixels. This results in a reduction of the read-out time and the read-out noise (as most X-rays will be confined to two $3 \times 3$ bins). The storage section is shielded from direct illumination by the housing. A thin Al light shield is put on top of each CCD to reduce the sensitivity to optical light. This light shield is isolated from the Si by a $\mathrm{MgF}_{2}$ isolation layer (about $26 \mathrm{~nm}$ thick). The $\mathrm{Al}$ shield is $75 \mathrm{~nm}$ thick for the two CCDs closest to the optical axis (short wavelength end of the bandpass), $68 \mathrm{~nm}$ for the next three CCDs, and $45 \mathrm{~nm}$ for the four CCDs furthest away from the optical axis. This gives a reduction in stray light between $10^{5}$ and $10^{2}$, equivalent to $1 \mathrm{e}^{-}$per pixel per readout (compared with the electronic noise of $5 \mathrm{e}^{-}$). The CCDs, operated in a just depleted mode, have an energy resolution of $\sim 160 \mathrm{eV}$ (FWHM) at $2 \mathrm{keV}$. This is sufficient for an efficient separation of the spectral orders.

\subsection{Readout and control electronics}

The electronic block diagram of the focal plane camera and its control electronics is shown in Fig. 4. It consists 


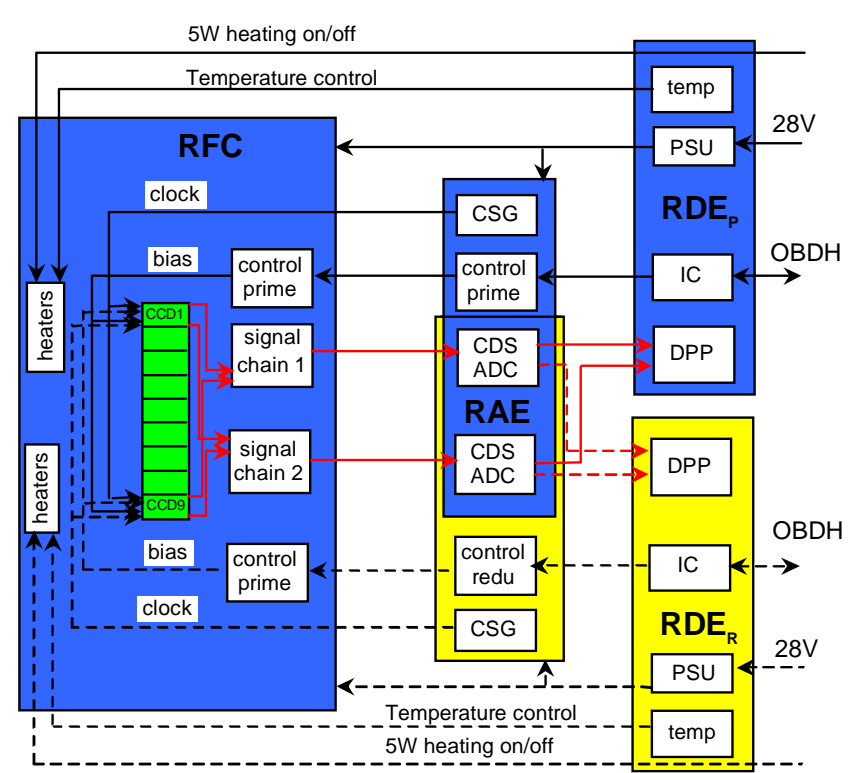

Fig. 4. Block diagram of RGS electronics. Cold redundancy is indicated by yellow and dashed lines, the signal chain (which is warm redundant) by red lines

of the focal plane camera proper (RFC), a unit containing the analog electronics (RAE) and two units (one redundant) with the digital electronics (RDE). The instrument is under control of the Instrument Controller (IC) which is fully programmable. The IC sets all bias settings of the CCDs and also configures the Clock Sequence Generator (CSG) which controls the clock drivers for each CCD. Different clock patterns can be loaded from the ground. The nominal readout of each CCD is through two output nodes resulting in different gains and offsets per read-out node of each CCD. The signals are amplified close to the focal plane camera and digitized in the RAE using a correlated double sampler (CDS). All data are then transferred to the Data Pre-Processor. The temperature of the CCD bench is controlled by the RDE and this unit includes also the power converter (Power Supply Unit). All interfaces with the spacecraft On Board Data Handling bus $(\mathrm{OBDH})$ are channeled via the IC. During periods when RGS is switched off (immediately following launch and during eclipses) the spacecraft provides a fixed power level to the CCD bench to avoid too much cooling.

The control chain is fully cold redundant (hence two RDEs) whereas the signal chain is warm redundant. In case of a failure in one of the two signal chains it is possible to read out the CCDs through one output node. In case of the failure of a single CCD the others will not be affected and the second camera will provide the required redundancy (although the effective area will then be reduced).

\subsection{Instrument modes}

Three operating modes are available within the RGS: SPECTROSCOPY, HIGH TIME RESOLUTION and DIAGNOSTIC. The most commonly used mode is
Table 2. Typical CCD parameters

\begin{tabular}{|rrl|}
\hline parameter & value & comment \\
\hline typical event size & 1.3 to 1.7 & pixels in $1 \times 1 \mathrm{OCB}$ \\
partial event fraction & $\sim 5 \%$ & $\mathrm{P}-\mathrm{K} \alpha ; 20 \%$ to $40 \%$ of $E_{\text {photo }}$ \\
$I_{\text {dark }}$ & 0.02 to 0.08 & $\mathrm{e}^{-} /$pixel $/ \mathrm{s}$ at $-80^{\circ} \mathrm{C}$ \\
hot columns RFC1 & 5 & at $-80^{\circ} \mathrm{C}$ \\
hot columns RFC2 & 1 & at $-80^{\circ} \mathrm{C}$ \\
$\mathrm{QE}$ & $>80 \%$ & for $E=0.35-2 \mathrm{keV}$, see Fig. 5 \\
$\mathrm{CTI}_{\text {parallel }} /$ transfer & $<10^{-5}$ & at $-80^{\circ} \mathrm{C}$ except near edges \\
$\mathrm{CTI}_{\text {serial }} /$ transfer & $\sim 310^{-5}$ & at $-80^{\circ} \mathrm{C}$ \\
$\mathrm{Si}$ thickness & $\sim 10 \%$ & variation over $\mathrm{CCD}$ \\
read-out noise & 5 to $6 \mathrm{e}^{-}$ & \\
\hline
\end{tabular}

SPECTROSCOPY: the nine CCDs are read out sequentially in frame transfer mode (with $3 \times 3$ on chip binning) resulting in an accumulation time of about $5.7 \mathrm{~s}$. After applying a low signal threshold, hot columns and hot pixels are rejected (defined by a lookup table). The remaining pixels are treated in a programmable processor (DPP). Pixels exceeding an upper signal threshold and pixels with too complex structures are rejected (these are due to cosmic rays), and only events which fit in a $2 \times 2$ pattern are transferred to the ground (including information on their shape). The HIGH TIME RESOLUTION mode affords the shortest accumulation time (about $15 \mathrm{msec}$ ) if only one CCD is read out. This is obtained by summing the image in the cross dispersion direction over 74 pixels, thus eliminating the possibility to use data cuts in the cross dispersion direction to reduce the background. More than one CCD can be used to collect data, but this increases the accumulation time accordingly. In the DIAGNOSTIC mode the on-board data processing is bypassed completely, and the full images are stored on board and transferred to the ground. These data are used for dark current and system noise level verification. Using the same mechanism as in the DIAGNOSTIC mode, full images can be transferred to the ground in SPECTROSCOPY mode as well, but at a low repetition rate.

\section{Ground calibrations}

The ground calibrations concentrated on the grating and CCD performance. These measurements were performed in three stages: first the individual gratings and CCDs were characterized; next we performed measurements after assembly of the reflection grating array and focal plane camera. Finally, end-to-end tests were performed with these units in combination with the mirrors. Prior to launch, the two RGS instrument chains went through their environmental tests (vibration, acoustic and thermal).

\section{1. $C C D$ response}

To select the flight devices from the larger set of available CCDs several diagnostic tests were performed. CCDs were singly installed in a dedicated camera which was connected to both a fluorescent X-ray source and a UV source. Most measurements were performed at $-80{ }^{\circ} \mathrm{C}$. 

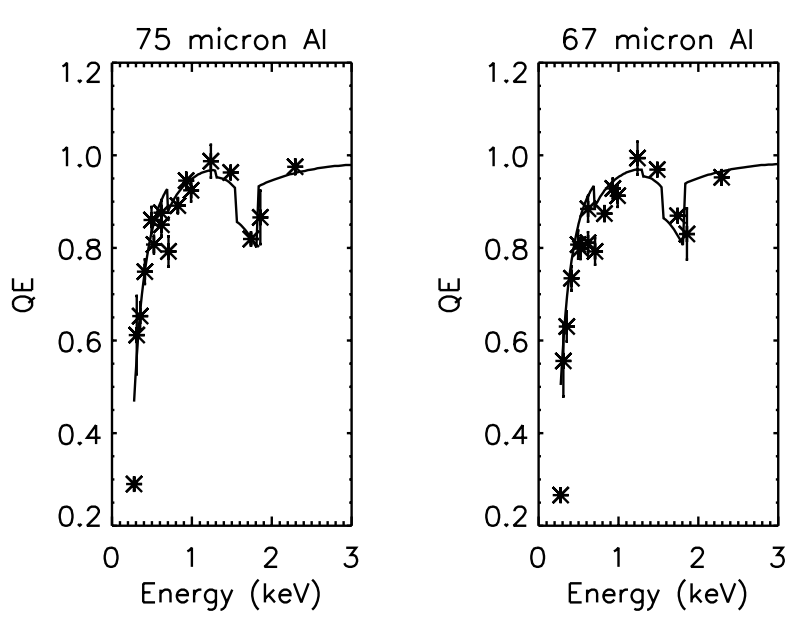

Fig. 5. Calculated and measured quantum efficiency for three typical devices

Where relevant, measurements were also performed at other temperatures (between $-60{ }^{\circ} \mathrm{C}$ and $-120{ }^{\circ} \mathrm{C}$ ).

Typical values for the CCDs are given in Table 2 where we also indicate whether a parameter is temperature dependent. Although the read-out noise is low, there is some fixed pattern noise induced by the electronics. This becomes visible if the low signal threshold is close to the offset, but the effect on the CCD energy resolution is negligible. To optimize the energy response of the CCDs they are operated such that the silicon is just depleted (the electrical field extends to the back side to reduce the diffusion of the charge over more pixels and thus minimizing the read-out noise for an X-ray which is spread over more pixels). Charge collection and readout is controlled by 14 bias voltages per CCD, and these are optimized to minimize the number of multiple pixel events. For $1 \times 1 \mathrm{OCB}$ this results in a typical event size of 1.5 pixels depending on the energy. Partial events are X-ray events that are absorbed close to the surface and therefore lose part of their charge in this surface layer, despite its passivation (van den Berg et al. 1996). This fraction, derived from the measured intensity between $20 \%$ and $40 \%$ of the energy of the absorbed X-rays, has been measured as function of position on the device and only devices with a sufficiently uniform backside and low partial event fraction have been selected. The Charge Transfer Inefficiency (CTI) is a measure for the charge loss when the pixel content is transfered from one pixel to the next and is caused by traps in the silicon. The CTI is in general very low (see Table 2) except near the edges of the CCDs where, possibly due to mechanical stress during production (dicing), the parallel CTI increases to $\sim 10^{-3}$. In Fig. 5 the modeled and measured quantum efficiency of two typical devices are shown. The data were collected during a long beam test at MPE with a beam that was normalized using an accurately calibrated Si drift chamber. Edges due to $\mathrm{Si}, \mathrm{Al}, \mathrm{Mg}$, and $\mathrm{F}$ are clearly visible. Additional measurements were performed at the Bessy synchrotron facility, in order to map the X-ray Absorption Fine Structure (variations up to a few \%, see Bootsma et al. 2000).

After the flight CCD's were selected, a number of additional measurements were performed on a few devices. The long term stability of the devices was verified with repeated UV and X-ray measurements over a period of four months on a single device that was kept continuously cooled and under vacuum. No degradation of the performance was observed. Using the long-term X-ray measurements the uniformity of the $\mathrm{Al}$ filter was verified. The X-ray transmission of the filter was found to be uniform within the accuracy of the measurement (1\% per pixel). One CCD has also undergone repeated thermal cycles to verify the stability and integrity of the $\mathrm{Al}$ filter.

Two devices were exposed to a proton spectrum representative of the exposure during a 10 year mission (consistent with $10^{9} 10 \mathrm{MeV}$ equivalent protons) under a $25 \mathrm{~mm}$ thick aluminum flight-representative housing. The parallel CTI degraded to $\sim 10^{-3}$ at $-80^{\circ} \mathrm{C}$ as expected. It was later verified that lowering the temperature to $-120^{\circ} \mathrm{C}$ reduces the effect of this degradation. Following the launch of Chandra the sensitivity of the RGS devices to soft protons was studied as this mission experienced a significant degradation of the CCD response in its early phase due to soft protons reflected off the mirrors. For the $30 \mu \mathrm{m}$ thick back illuminated devices, protons with energies of 1.4 to $1.5 \mathrm{MeV}$ cause maximum damage in the CCD channels. Additional tests were performed at these energies. It was found that the CTI degrades to $210^{-3}$ for a $10^{7}$ dose of 1.4 to $1.5 \mathrm{MeV}$ protons. Fortunately, the in orbit spectrum of protons is steep. Furthermore, protons "focused" by the mirrors, must be reflected off the gratings before they hit the CCDs, which decreases the flux incident on the cameras further. Based on these results the risk to the CCDs in the RGS configuration was considered minimal.

The CCD response has been modeled based on the measurements from the end-to-end ground calibration tests, where the gratings provided almost monochromatic illumination. The model is based on a set of physical parameterizations of the absorption and charge collection processes. The absorption probability is calculated in the standard way by using the Henke atomic constants (Henke et al. 1993). The charge collection efficiency is modeled as an exponential function of the depth into the device. We add a noise component, which includes Fanonoise and electronic readout noise. Convolving these three components analytically yields a model with only two adjustable parameters: the electronic noise and the effective charge collection depth, $\rho$. By using the ratio of $\rho$ over the mean absorption length these can be reduced to a single energy independent parameter. Assuming a heuristic Fano factor of 0.14 , the electronic noise was found to be 5 to 6 electrons (using $1 \times 1 \mathrm{OCB}$ ), which is consistent with our measurements. The pulse height distribution of this model is compared to data in Fig. 6. The energy resolution of the CCD's is used to separate the orders of the dispersed spectra. Order selection filters are based on the modelled CCD reponse, and the accuracy of this model affects the 


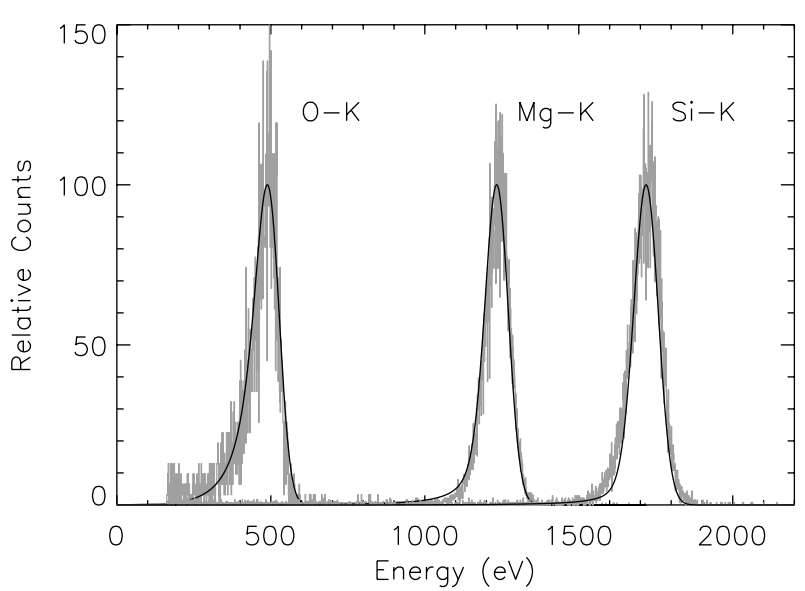

Fig. 6. Composite pulse height spectrum for $\mathrm{O}-\mathrm{K} \alpha, \mathrm{Mg}-\mathrm{K} \alpha$ and $\mathrm{Si}-\mathrm{K} \alpha$ (histogram) as well as the modeled response (dark smooth curve)

estimate of the integral of the selected distribution. This in turn is used for the calculation of the effective area. The effect of inaccuracies in this model on the effective area is estimated to be of the order of $2 \%$ for filters that include $>90 \%$ of the distribution.

\subsection{Reflection grating response}

The grating replicas were selected based on quantitative measurements of several properties. The following measurements, performed for every grating, were obtained at a dedicated facility at Columbia University Nevis Laboratories (Craig et al. 1988). The results were used to reject and remanufacture parts, and to ultimately define two nearly identical sets that were used to complete the two grating arrays (Rasmussen et al.1998).

- Grating figures were measured using a Zygo interferometer with the four grating corners constrained to a plane;

- Grating efficiencies were measured in $m=0, m=-1$ and $m=-2$ for the nominal incidence angle at five energies: $\mathrm{Al} \mathrm{K} \alpha(8.34 \AA), \mathrm{Cu} \mathrm{L} \alpha(13.34 \AA), \mathrm{Fe} \mathrm{L} \alpha$ $(17.6 \AA \& 20.1 \AA)$, and $\mathrm{O} \mathrm{K} \alpha(23.6 \AA)$;

- Grating surface microroughnesses that redistribute light on small angular scales ( $\sim 1$ arcmin) were quantified by measuring the two dimensional scatter distribution at $\mathrm{Al} \mathrm{K}(8.34 \AA)$ in $m=0$, for the nominal incidence angle and geometry.

The orientation and twist of the gratings were measured interferometrically as assembled with a common reference coordinate. This, and the individual figure measurements provides the optical figure of the assembly as a whole. The composite grating array figures were verified after the mating of the grating arrays to the XMM mirror modules, using a parallel UV beam at the Centre Spatial de Liège (Stockman et al. 1998).

In addition to the measurement list above, the following measurements were performed on a subset of

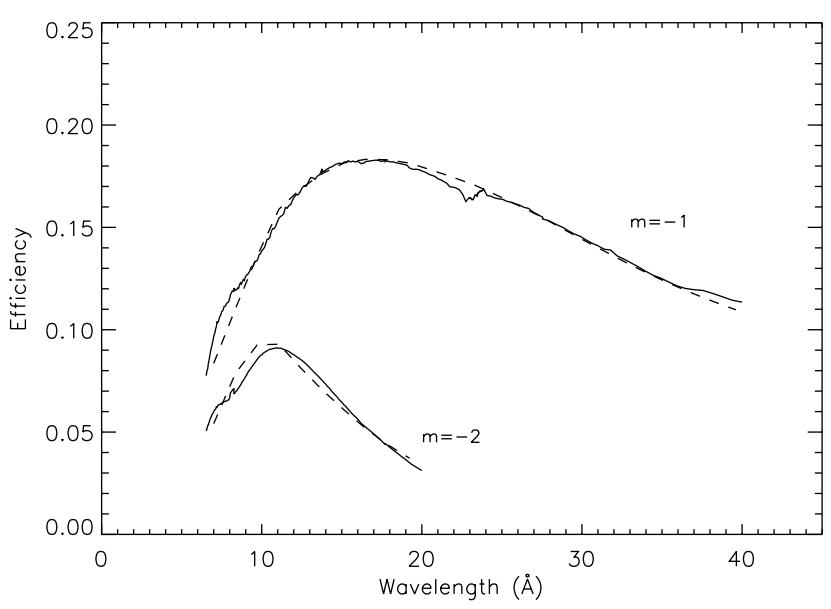

Fig. 7. Efficiencies for first and second order as measured (solid curve) and as modeled (dashed curve)

gratings that helped constrain physical modelling of the RGS; in particular the grating reflectivity. These measurements were performed randomly on one grating in every six, unless otherwise noted:

- Four gratings were measured for their reflectivity functions in $m=0, m=-1$ and $m=-2$ between 7 and $80 \AA$ and the nominal incidence angle using a monochromatized beamline at the BESSY synchrotron light source;

- The diffraction efficiencies for orders 0 through -5 were measured at $\mathrm{Cu} \mathrm{L}(13.34 \AA)$;

- The $m=0$ efficiency function was measured over the $1.5-2.5^{\circ}$ incidence angle range, which is sensitive to details of the groove profile;

- The scatter distribution in $m=-2$ for $\mathrm{Al} \mathrm{K}$ $(8.34 \AA)$ which further constrains the microroughness parameters.

The reflection grating response is a combination of the measured geometric quantities (the composite array figure) and a physical model of the grating.

A model of the diffraction efficiency was constructed using the full vector solution to Maxwell's equations subject to the boundary conditions of the grating profile. A scalar theory modification was implemented to account for coherent and incoherent modulations on the surface of the grating (Cottam et al. 2001). The parameters of the model were determined by iteratively fitting simultaneously calibration data from the long-beam and BESSY facilities. An incoherent noise component that scatters light to large angles and out of the standard measurement apertures was characterized by fitting inter-order scans taken at the Nevis long-beam facility. The measured efficiency is predicted by integrating the normalized line profile with both small and large angle scattering components over the angular range subtended by the measurement aperture. The model is consistent with the measurements to $\sim 5 \%$ for all incident angles, spectral orders, and wavelengths above $9 \AA$. This is illustrated in Fig. 7, where the results 


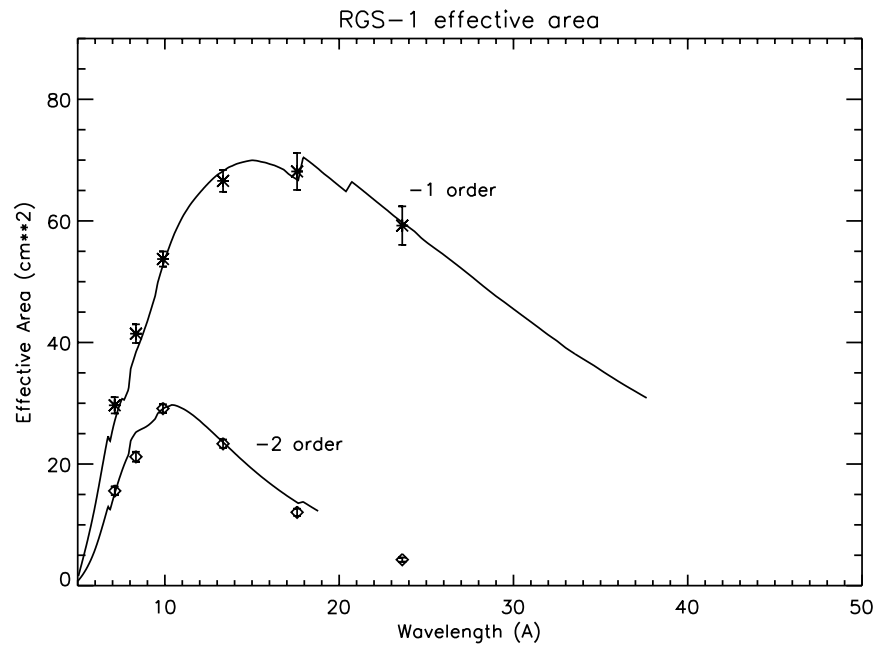

Fig. 8. Measured and modelled effective area for RGS1 (corrected for finite source distance)

of the reflectivity measurement for one grating at Bessy is shown.

\subsection{End-to-end calibrations}

The X-ray performance was verified at the MPE long beam "Panter" facility (Aschenbach et al. 1979). Due to the finite source distance $(120 \mathrm{~m})$ at this facility, the spectrometer does not focus properly if the optical elements and detectors are positioned in the flight configuration. In particular, the focus of the telescope moves outward $500 \mathrm{~mm}$ relative to the nominal focus. To maintain the Rowland circle, the RGA must be moved by the same amount. Owing to the finite width of the X-ray lines generated with an electron impact target source, the resolution properties could not be verified directly for the longer wavelength portion of the RGS band. However, using higher order spectra of short wavelength lines, it was still possible to investigate all of the various contributions to the line spread function (Rasmussen et al. 1998). These measurements indicated that the two RGAs have slightly different focussing properties. This explains the different expectations for the resolution as is given in Fig. 11.

The effective area was calibrated at the Panter facility at 6 energies, on axis. The off-axis response was calibrated at one energy $(\mathrm{Al}-\mathrm{K} \alpha)$. Given the finite source distance, the telescope and RGA are under-illuminated, and the measured effective area had to be corrected for this effect using a detailed raytrace model of this configuration. These corrections were verified using segmented illumination of the mirrors ( 16 azimuthal sectors $\times 4$ radial selections). The results of the end-to-end effective area calibration agreed with model calculations to within $5 \%$ for both the first and second spectral orders. This is illustrated in Fig. 8 .
Table 3. Main calibration targets

\begin{tabular}{|ll|}
\hline object & purpose \\
\hline Capella & $\begin{array}{l}\text { wavelength scale, bore sight, } \\
\text { effective area, CCD response }\end{array}$ \\
HR 1099 & wavelength scale, CCD CTI \\
AB Dor & repeated wavelength scale; after June \\
3C 273 & on- and off-axis effective area \\
GX 13+1 & effective area $(\lambda<10 \AA$ ) \\
Mkr 421 & on-axis effective area \\
Lockman hole & background estimate \\
PKS 2155-304 & effective area simultaneously with Chandra \\
Sco X-1 & CCD gain and CTI (partially successful) \\
Canopus & stray light \\
\hline
\end{tabular}

\section{In-orbit performance}

The commissioning phase of the XMM-Newton instruments started on January 4, 2000, followed by a calibration and performance verification phase between March 9 and July 4, 2000. During these periods a number of astrophysical targets were observed (see Table 3 ).

After the first week of operations, an electronic component in the clock driver of CCD 4 in RGS2 failed. A similar problem showed up in early September, 2000, on CCD 7 of RGS1. The affected wavelength ranges are 20.1-23.9 for RGS2 and 10.5-14 $\AA$ for RGS1 reducing the effective area by a factor of 2 for these ranges. The operational conditions have been modified to reduce the load of these commonly used components to a minimum.

An example of the data is shown in Fig. 9, where the dispersed image of Capella is shown in the upper panel, while in the lower panel CCD pulse height is plotted against dispersion. In the lower panel the first, second, and even higher orders are visible. The four internal calibration sources (at $1487 \mathrm{eV}$ and at $677 \mathrm{eV}$ ) are also discernible. In order to extract a spectrum from these data, the following cuts are applied. In the cross-dispersion direction a filter is applied which includes typically $90 \%$ of the total intensity. The spectral order is selected in the CCD pulse height versus dispersion angle space (lower panel of Fig. 9) with a joint pulse height-dispersion filter. The extracted spectra are shown in Fig. 10 and these are discussed in detail by Audard et al. (2001). In addition the exposure time per wavelength bin is calculated taking into account the data selections, rejected columns and pixels (either due to cosmics or to cosmetic blemishes in the CCD) and the dead space between CCDs (see also Erd et al. 2000).

\section{1. $C C D$ response}

A number of key performance parameters were checked. The gains of the CCDs were calibrated using the internal calibration sources and a few strong astrophysical continuum sources. The gains are slightly different from pre-flight but have been very stable since launch. The CCD energy resolution was verified with a few known emission lines. No changes with respect to the preflight 

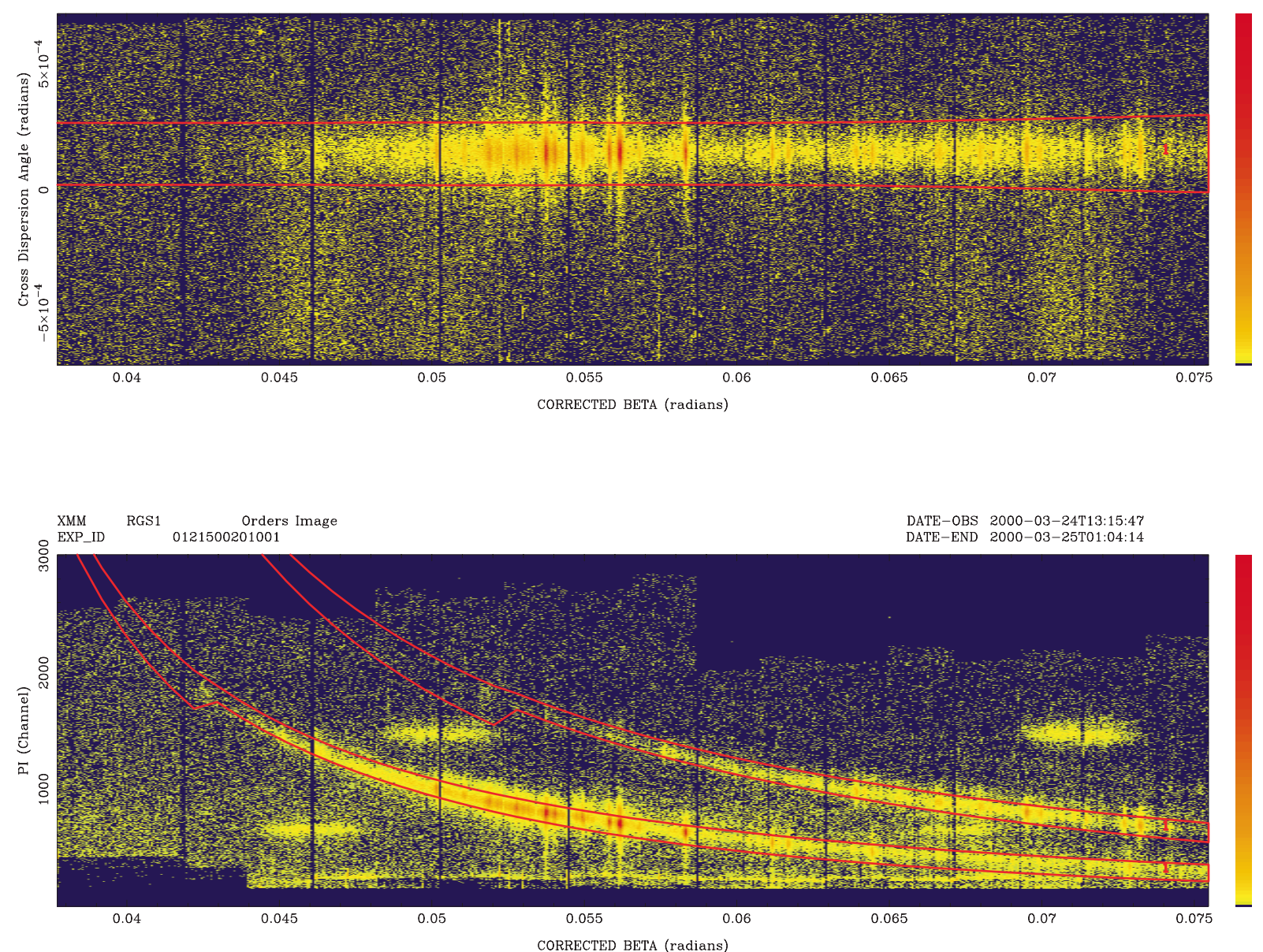

Fig. 9. Capella observation (id: 0121500201001). The color scale represents a logarithmic intensity scale. The dispersion axis runs horizontally and increases to the right. In the top panel the cross dispersion is along the vertical axis whereas in the bottom panel this is replaced by the CCD energy. In the bottom panel the low and high level thresholds are visible. Standard data selections are indicated by the red curves

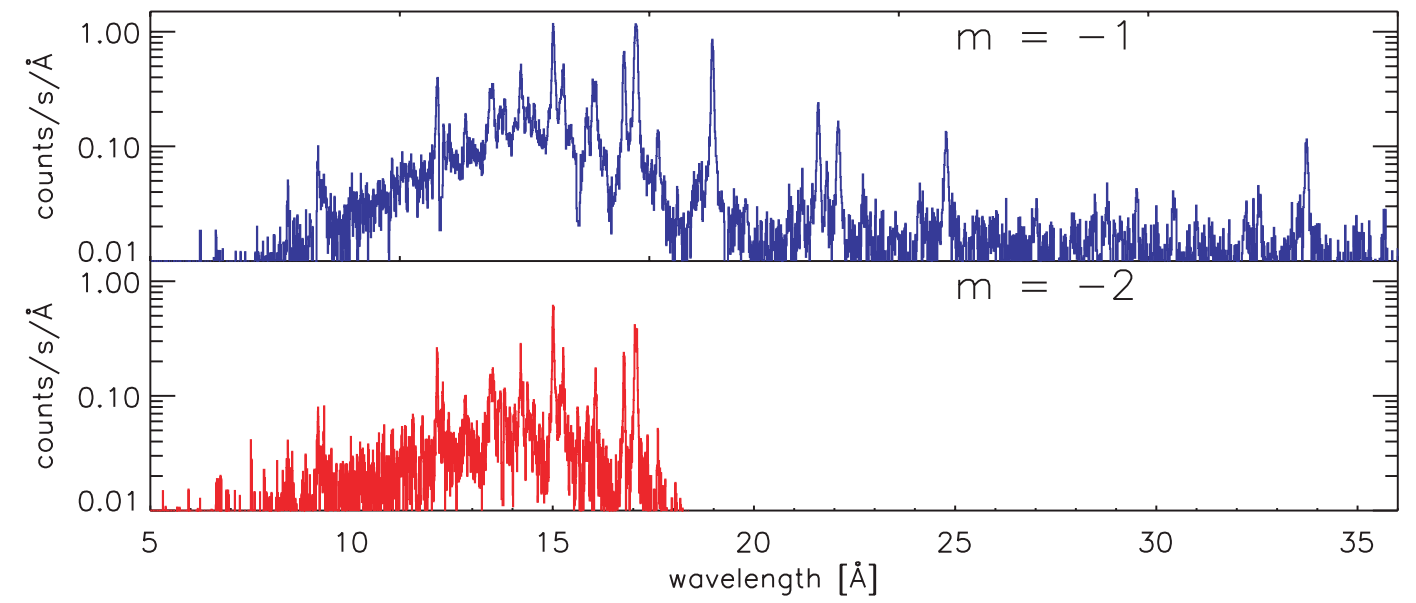

Fig. 10. First and second order spectrum for Capella observation. Emission lines are clearly visible (see Audard et al. 2000 for a full analysis)

conditions were observed. Position dependent offsets of the CCD energy scale due to straylight were measured and are consistent with the pre-launch expectations. Using DIAGNOSTIC data it was verified that the system peak (a combination of electronic noise, dark current and optical load of the CCDs) is very stable over an orbit (within $1 \mathrm{eV}$ ) with the exception of a jump of about $2 \mathrm{eV}$, which occurred following a very strong solar flare on July 7,2000 . The only component that is slightly variable are the hot pixels and columns. 
Special attention was given to a potential increase of the CTI due to soft protons (entering through the mirror) and hard protons (the mission is close to the solar max). This property of the system is monitored continuously. Based on these results (position of the $\mathrm{Al}$ and $\mathrm{F}$ peak as function of number of orbits) the maximum CTI increase is estimated to be $11^{-7}$ per transfer per orbit. Accepting a CTI loss of $25 \%$, which still allows for good order separation, this translates into a lifetime of more than 15 years without the need to lower the camera temperatures. Using the intensity of the internal sources it is possible to monitor contamination. Comparison of these intensities with the intensities from the ground calibration indicate an upper limit to any change in quantum efficiency $(\mathrm{QE})$ at the O-K edge of $8 \%$.

The CCDs also exhibit low amplitude, low pulse height Fixed Pattern Noise. This is a minor effect, easily suppressed with an appropriate pulse height filter.

\subsection{Line spread function}

The Line Spread Function (LSF) is a convolution of the mirror response and the grating response, and it depends on the proper alignment of these units. The mirror response includes the figure properties and co-alignment of all 58 nested mirrors. The grating response depends on the co-alignment and flatness of the 182 gratings that are integrated per RGA. In addition there is also a significant (but well calibrated) component due to scattering on the surfaces of the mirrors and the gratings (seen as the horizontal distribution of events in the bottom panel of Fig. 9). The various components scale differently with wavelength, which gives rise to a composite line shape which can not easily be characterized in terms of a simple analytical function. The shape of the core largely determines the ability of the spectrometer to separate closely spaced emission lines. The telescope blur contributes a constant term to the spectrometer line width, while misalignments and flatness errors of the gratings contribute a term which slowly increases with increasing wavelength. The scattering component is most significant at the shortest wavelengths and the highest diffraction orders. This is illustrated in Fig. 11 where the predicted resolution for the two RGSs is shown (the steep rise below $7 \AA$ is due to the scattering component of the gratings). Also shown are the measurements, which are deduced from a few narrow, bright emission lines in HR 1099 (Ly $\alpha$ lines of $\mathrm{Ne}$ $(m=-1,-2), \mathrm{O}, \mathrm{N}$ and $\mathrm{C})$. This demonstrates that the instrument resolving power is close to the expectations. The explicit spectrometer line response is close to predictions based on a raytrace model for the spectrometer.

The ability to detect weak emission lines above the background or the continuum is better measured by the half energy width $(H E W)$ of the profile. This width, especially at short wavelengths, is more dependent on the amplitude and width of the scattering wings. Again, the data agree with the predictions and in the $H E W$

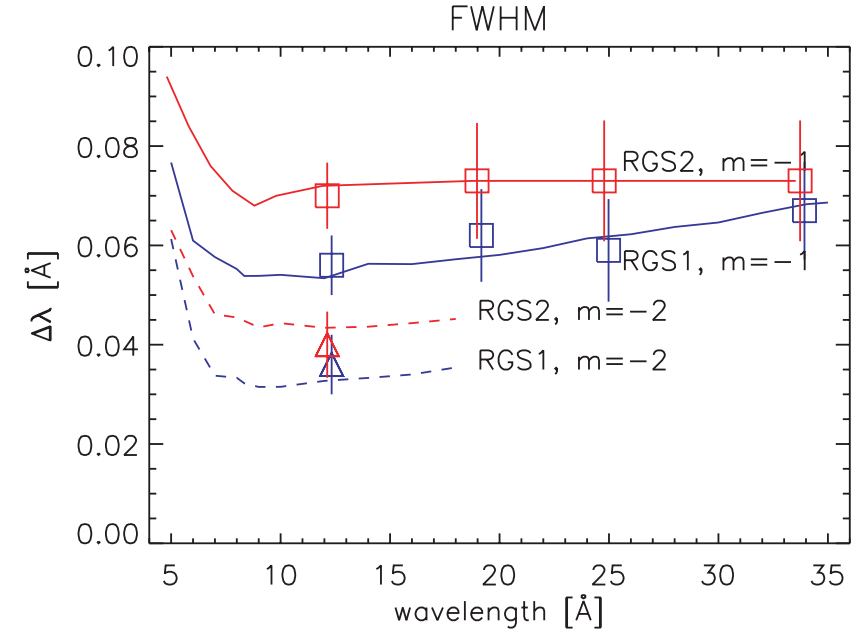

Fig. 11. Comparison of the measured and predicted resolution (FWHM) for RGS1 (red) and for RGS2 (blue). Data for second order are shown as dashed lines and triangular datapoints

sense the resolving power goes to $\sim 800$ at the longest wavelengths.

\subsection{Wavelength calibration}

To measure wavelengths, event locations (pixel coordinates) are mapped into 3-dimensional coordinates using the camera geometry measured at $20^{\circ} \mathrm{C}$ and a thermalmechanical model for the optical bench. The dispersion angle $\beta$, and the polar angle $\xi$ subtended from the mirror in the cross-dispersion direction, are then assigned based on the spacecraft geometry. The angle of incidence of a photon on the grating and the dispersion angle determine the measured wavelength according to the dispersion equation Eq. (1). The relative misalignment between the RGS instrument axis and the star tracker axis (the bore-sight described by three Euler angles), the rotation of the grating array relative to the RGS axis $\left(R_{y}(\mathrm{RGA})\right)$, and the position of the focal plane camera $\left(T_{z}(\mathrm{RFC})\right)$ are used to calculate $\alpha$ and $\beta$.

In-flight calibrations further refined ground-based measurements of these geometric parameters. There is a inherent degeneracy in the determination of these five parameters, however, so $R_{y}(\mathrm{RGA})$ was fixed to its ground alignment value. Using on-axis and off-axis (up to 10 arcmin) observations of Capella, HR 1099, and AB Dor, we constrained the other four parameters using the theoretical wavelengths of $\mathrm{Ly} \alpha$ lines of $\mathrm{Mg}, \mathrm{Ne}, \mathrm{O}, \mathrm{N}$, and $\mathrm{C}$. The geometric parameters were consistent with expected tolerances for these parameters from the ground measurements. The measurement of wavelengths with RGS is currently accurate to $8 \mathrm{~m} \AA$ (rms) across the entire wavelength band, based on the distribution of the wavelength residuals of the calibration lines.

The boresight angles as well as the cross-dispersion position of the focal plane camera $\left(T_{y}(\mathrm{RFC})\right)$ also give the angle $\xi$. It was found to be consistent with the crossdispersion measurements to better than 4 arcsec (rms). 


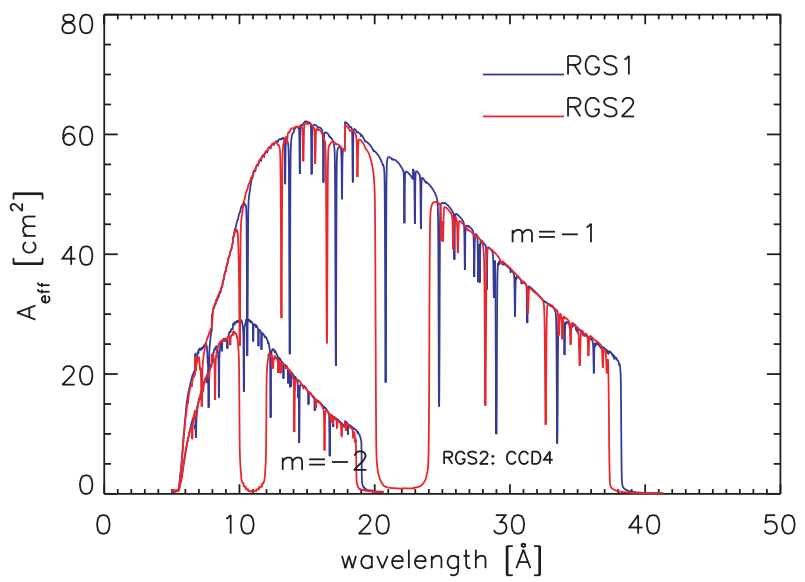

Fig. 12. Effective area for the two RGSs (clearly visible are the gaps between the CCDs and the failing read-out of CCD4 in RGS2, the narrow drops in the curves are due to corrections for cosmetic blemishes)

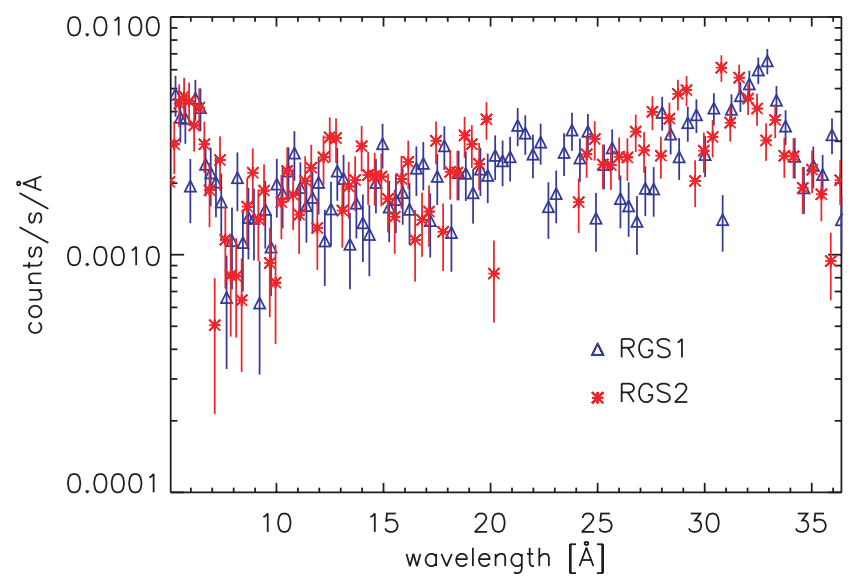

Fig. 13. Typical countrate for standard data selections $(m=$ $-1,90 \%$ in CCD pulse height, full range in cross dispersion direction for a quiet part of the Lockman Hole observation

This can be used to predict the measured position of the source and is used in the calculation of the instrument response.

\subsection{Effective area}

The effective area model of the RGS has been verified during in-flight calibration. Off-axis observations were performed on the continuum sources 3C 273 and GX 13+1, which sample both the full wavelength band and the short wavelength region, respectively. The ratios of the observed count rates in the off-axis to on-axis measurements were compared to the ratios predicted by the effective area model. This comparison is sensitive to the details of the model components, particularly to the grating efficiency and the geometric intercept and vignetting factors. The measured behavior agrees with the predicted behavior to within the statistical uncertainties for off-axis angles up to 10 arcmin in both first and second order, for all wavelengths above $9 \AA$. As a further check of the model the second to first order count rate ratio for both sources was compared with the predicted ratio. The prediction agrees across the band with the exception of the 7 to $9 \AA$ region, where the ratio is overpredicted.

There is evidence for a narrow absorption feature at the neutral $\mathrm{O} \mathrm{K}$ edge. It has a a threshold wavelength of $23.5 \AA$, and a relative amplitude of about $27 \%$. This feature appears in all bright continuum sources. A comparison of the spectrum of the bright BL Lac object PKS 2155-304 as measured with the RGS, and with the Chandra LETGS suggests that the feature is instrumental. A detailed calibration is in progress.

The absolute effective area of the RGS will be crosscalibrated with the diffraction grating spectrometers on Chandra, as well as with the Chandra ACIS and XMMNewton EPIC detectors, using suitable bright cosmic sources. This work is currently in progress.

The effective area for the nominal data selections is shown in Fig. 12 for the two RGSs. Due to the data selections the maximum effective area is lower than measured during the end-to-end tests (see Fig. 8 for reference).

\subsection{Instrument background}

The background model for the RGS was cross-checked using a deep observation of the Lockman Hole. Several different components contribute, including:

- minimum ionizing particles (protons and ions). Most of these particles deposit an energy outside the 0.35 to $2.5 \mathrm{keV}$ energy band and can thus easily be rejected;

- low energy electrons which enter through the telescope. Up to $20 \mathrm{keV}$ these are rejected by the electron deflector at the exit plane of the mirrors. Higher energy electrons create secondary radiation in the spacecraft and detector shielding;

- fluorescence lines in the detector housing due to interactions with the electrons and other minimum ionizing particles. The expected strong fluorescence from the detector housing material, $\mathrm{Al}$, is strongly suppressed by a $\mathrm{Au}$ coating. One still expects to see $\mathrm{Al} \mathrm{K} \alpha$ and $\mathrm{Au}-\mathrm{M}$ emission in the RGS energy band. These lines are indeed observed at a very low intensity (about $10^{-3}$ counts $/ \mathrm{cm}^{2} / \mathrm{s}$ for $\mathrm{Au}$ and $410^{-4}$ counts $/ \mathrm{cm}^{2} / \mathrm{s}$ for $\left.\mathrm{Al}\right)$;

- soft protons entering through the mirrors: these protons are focussed to the detectors by scattering on the mirrors and gratings. This results in a beam centered at the zero order position, with a very large width in the dispersion direction. An analysis of the background observed in the Lockman Hole data yields a typical Gaussian distribution with a $5.8^{\circ}$ and $0.3^{\circ}$ width in the dispersion and cross dispersion direction respectively. This component, including the width in the dispersion direction, is clearly time variable;

- calibration sources: these can each be modelled as a two dimensional Gaussian intensity distribution with a typical width of 78 pixels $(3 \times 3$ OCB $)$ in the 
Table 4. Key performance parameters

\begin{tabular}{|rrl|}
\hline parameter & value & comment \\
\hline$A_{\text {eff }}$ & $140 \mathrm{~cm}^{2}$ & peak effective area \\
$\lambda$ & $6-38 \AA$ & wavelength range first order \\
$\lambda / \Delta \lambda(F W H M)$ & $100-500$ & resolution (line separation) \\
$\lambda / \Delta \lambda(H E W)$ & $100-800$ & resolution (weak line detection) \\
$\delta \lambda$ & $8 \mathrm{~m} \AA$ & wavelength accuracy \\
\hline
\end{tabular}

dispersion direction and 37 pixels in the cross dispersion direction. The fraction spilling into the standard event selection regions is very small, since these are offset in the cross dispersion direction;

- read-out noise: although strictly speaking this is not a background component, the noise characteristic of each CCD has a tail which cannot be distinguished from proper X-rays.

Following the onboard background rejection, the major constituents of the background are a tail on the CCD response due to readout noise, and the soft proton radiation. Using a model for the behavior of these components, the Lockman Hole data appear to be adequately described. However, as some of the components are clearly time variable, it is not trivial to use the same model for other observations of extended sources. For point sources, however, one can simply select a region on the CCDs in the cross dispersion direction and use the same windows in the CCD pulse height as for the source. For reference we give the first order spectrum for a standard selection (90\% window in CCD energy only) of the Lockman Hole data for a quiet period (see Fig. 13). The increase in background below $8 \AA$ is due to a change in the width of the pulse height filter at that wavelength (given as red curves in Fig. 9).

\section{Conclusion}

The RGS performs as expected with the exception of the electronics of two CCDs. Several papers in this issue attest to its unique capabilities. Simultaneous measurements together with the three EPIC cameras and the Optical Monitor enhance the scientific return significantly. Although some small refinements in the performance parameters can be expected following analysis of a larger dataset, the key parameters as listed in Table 4 are characteristic.
Acknowledgements. Based on observations obtained with $X M M$-Newton, an ESA science mission with instruments and contributions directly funded by ESA Member States and the USA (NASA). We are particular grateful to Mr. Robert Lainé and his team for their support during the development of the hardware. The Laboratory for Space Research Utrecht is supported financially by NWO, The Netherlands Organization for Scientific Research. The Mullard Space Science Laboratory acknowledges financial support form the UK Particle Physics and Astronomy Research Council. The Swiss contribution has been supported by the PRODEX program, the Swiss National Science Foundation (Grant 21-49343.96), and the Swiss Commission for Space Research. The Columbia and Lawrence Livermore groups are supported by the U.S. National Aeronautics and Space Administration.

\section{References}

Aschenbach, B., Bräuninger, H., Stephan, K-H., \& Trümper, J. 1979, Proc. SPIE, 183, 234-238

Audard, M., Behar, E., Güdel, M., et al. 2001, A\&A, 365, L329 Berg, M. L., van den, Boggende, A. J. F., den, Bootsma, T. M. V., et al. 1996, Nucl. Instr. Meth., A 377, 312

Bootsma, T. M. V., Zwet, E. J., van Brinkman, A. C., et al. 2000, Nucl. Instr. Meth., A 439, 575-581

Brinkman, A. C., Aarts, H., Boggende, A., et al. 1998, The Reflection Grating Spectrometer on XMM, Proc. of First XMM Workshop, www.vilspa.esa.es

Cottam, J., et al. 2001, in preparation

Craig, W., Hailey, C., Morales, R., et al. 1988, Proc. SPIE, 982,362

Erd, C., Audard, M., Boggende, A. J. den, et al. 2000, Proc. SPIE, 4140

Henke, B. L., Gullikson, E. M., \& Davis, J. C. 1993, Atomic Data and Nuclear Data Tables, 54, 181

Jansen, F., Lumb, D., Altieri, B., et al. 2001, 365, L1

Kahn, S. M. 1990, in High Resolution X-ray Spectroscopy of Cosmic Plasmas, ed. P., Gorenstein \& M. Zombeck (Cambridge, Cambridge University Press)

Kahn, S. M., Cottam, J., Decker, T. A., et al. 1996, Proc. SPIE, 2808,450

Rasmussen, A. P., et al. 1998, Proc. SPIE, 3444, 327

Stockman, Y., Domken, I., Hansen, H., et al. 1998, Proc. SPIE, 3445,62

Thomsen, K. 1991, 4th European symposium on space environmental control systems, Florence, 21-25 Oct. 1991

Wilke, P. S., Decker, T. A., \& Hale, L. C. 1995, Proc. SPIE, 2445, 2 